\title{
Loss-of-function mutations in the glutamate transporter SLC1A1 cause human dicarboxylic aminoaciduria
}

\author{
Charles G. Bailey, ${ }^{1}$ Renae M. Ryan, ${ }^{2}$ Annora D. Thoeng, ${ }^{1}$ Cynthia Ng, ${ }^{1}$ Kara King, ${ }^{1}$ \\ Jessica M. Vanslambrouck, ${ }^{1}$ Christiane Auray-Blais, ${ }^{3}$ Robert J. Vandenberg, ${ }^{2}$ \\ Stefan Bröer, ${ }^{4}$ and John E.J. Rasko ${ }^{1,5}$
}

\begin{abstract}
${ }^{1}$ Gene and Stem Cell Therapy Program, Centenary Institute, Camperdown, New South Wales, Australia. ${ }^{2}$ Department of Pharmacology, University of Sydney, Sydney, New South Wales, Australia. ${ }^{3}$ Service of Genetics, Department of Pediatrics, Université de Sherbrooke, Sherbrooke, Quebec, Canada. ${ }^{4}$ Research School of Biology, Australian National University, Canberra, Australian Capital Territory, Australia. ${ }^{5}$ Cell and Molecular Therapies, Royal Prince Alfred Hospital, Camperdown, New South Wales, Australia.
\end{abstract}

\begin{abstract}
Solute carrier family 1, member 1 (SLC1A1; also known as EAAT3 and EAAC1) is the major epithelial transporter of glutamate and aspartate in the kidneys and intestines of rodents. Within the brain, SLC1A1 serves as the predominant neuronal glutamate transporter and buffers the synaptic release of the excitatory neurotransmitter glutamate within the interneuronal synaptic cleft. Recent studies have also revealed that polymorphisms in SLC1A1 are associated with obsessive-compulsive disorder (OCD) in early-onset patient cohorts. Here we report that $S L C 1 A 1$ mutations leading to substitution of arginine to tryptophan at position $445(\mathrm{R} 445 \mathrm{~W})$ and deletion of isoleucine at position 395 (I395del) cause human dicarboxylic aminoaciduria, an autosomal recessive disorder of urinary glutamate and aspartate transport that can be associated with mental retardation. These mutations of conserved residues impeded or abrogated glutamate and cysteine transport by SLC1A1 and led to near-absent surface expression in a canine kidney cell line. These findings provide evidence that SLC1A1 is the major renal transporter of glutamate and aspartate in humans and implicate SLC1A1 in the pathogenesis of some neurological disorders.
\end{abstract}

\section{Introduction}

Dicarboxylic aminoaciduria (DA; OMIM 222730) involves a striking excretion of urinary glutamate and aspartate, resulting from the incomplete reabsorption of anionic amino acids from the glomerular filtrate in the kidney. DA is an autosomal recessive disorder with an estimated frequency of 1:36,000 (1), which was first described in 1974 (2). The clinical features of the disorder have not been widely explored, due to the paucity of clinical cases; however, of the 4 independent cases examined, 2 report an association with mental retardation $(2,3)$. Solute carrier family 1 , member 1 (SLC1A1), also referred to in human studies as excitatory amino acid transporter 3 (EAAT3) or in rodents as excitatory amino acid carrier 1 (EAAC1), is a high-affinity anionic amino acid transporter expressed in the kidney, a wide variety of epithelial tissues, brain, and eye. Convincing studies in rabbits and rodents have established that SLC1A1 plays a major role in the renal and intestinal transport of glutamate and aspartate (4-6). SLC1A1 is also found in neurons throughout the cortex, particularly in the hippocampus, basal ganglia, and the olfactory bulb (7), in which it is thought to be involved in facilitating $\gamma$-aminobutyric acid and glutathione biosynthesis $(8,9)$. SLC1A1 functions to buffer local glutamate concentrations at excitatory synapses in the hippocampus and modulates the differential recruitment of glutamate receptor subtypes at extrasynaptic sites (10). To date, no germline mutations in SLC1A1 have been functionally characterized.

The study of the genetics and pathophysiology of aminoacidurias have guided our understanding of epithelial transport

Conflict of interest: The authors have declared that no conflict of interest exists. Citation for this article: J Clin Invest. 2011;121(1):446-453. doi:10.1172/JCI44474. physiology. Our group, through the Australian Aminoaciduria Consortium, and others have identified the genetic determinants of 4 out of the 5 principal aminoacidurias (11), including cystinuria (OMIM \#220100; type A SLC3A1, ref. 12, and type B SLC7A9, ref. 13); lysinuric protein intolerance (OMIM \#222700; SLC7A7, refs. 14 and 15); Hartnup disorder (OMIM \#234500; SLC6A19, refs. 16 and 17); and iminoglycinuria (OMIM \#242600; SLC36A2 and SLC6A20, ref. 18). DA has until now remained without a molecular explanation. Here we describe the first molecular characterization to our knowledge of human DA pedigrees. Affected family members inherited homozygous loss-of-function mutations (I395del and R445W) in SLC1A1. We demonstrate the potential pathogenic consequences of the mutations, using biochemical analysis after Xenopus laevis oocyte expression and subcellular localization studies.

\section{Results}

Probands were (proband 1) a 44-year-old Australian man with Italian ancestry and (proband 2) a 4-year-old girl of French-Canadian descent. Proband 1 presented with kidney stones at the age of 20, which also occurred in his brother at the same age. He was initially diagnosed with DA by urinary analysis, and persistent DA was confirmed at age 44 in this study. Proband 1 admitted to lifelong behavior comprising hand washing approximately 20 times per day and regularly rechecking the kitchen oven after leaving the house, both suggestive of an obsessive-compulsive trait. However, he declined formal psychological assessment for obsessive-compulsive disorder (OCD). Proband 2 was diagnosed with DA at birth in the Quebec neonatal mass urinary screening program (1), and persistent DA was confirmed at age 4 in this study. 


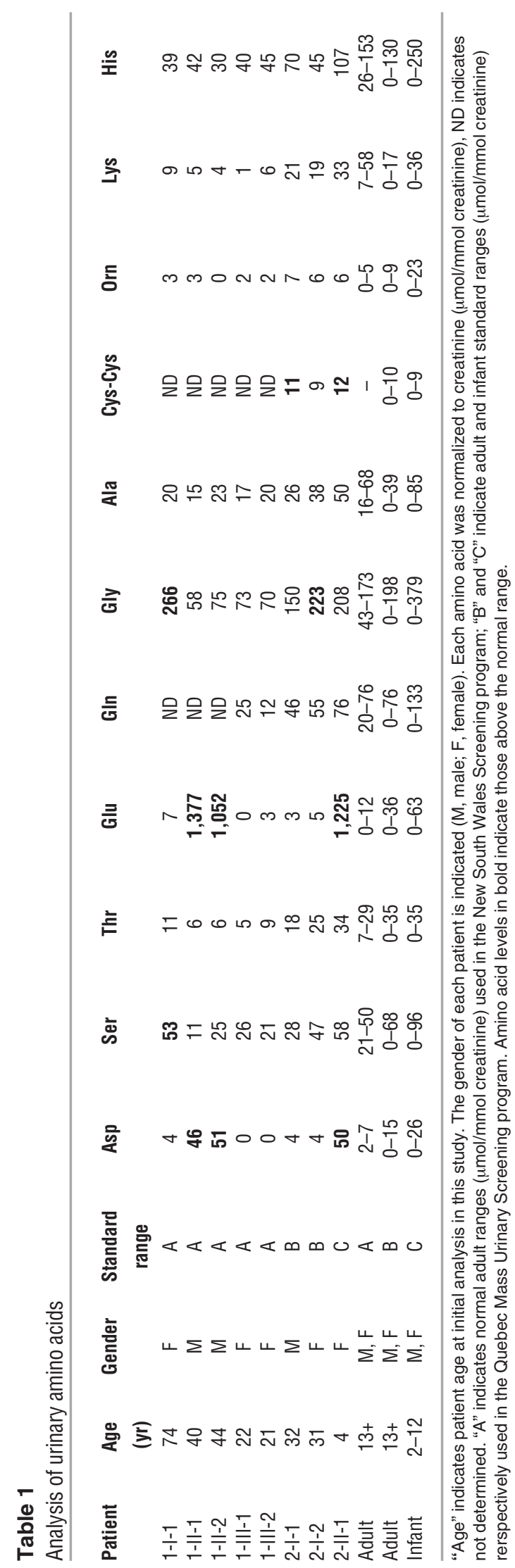

In proband 1 (patient 1-II-1), we confirmed elevations in the urinary glutamate and aspartate levels, with a urinary glutamate level of $1,377 \mu \mathrm{mol} / \mathrm{mmol}$ creatinine (normal range, $0-12 \mu \mathrm{mol} / \mathrm{mmol}$ creatinine) and an aspartate level of $46 \mu \mathrm{mol} / \mathrm{mmol}$ creatinine (normal range, $2-7 \mu \mathrm{mol} / \mathrm{mmol}$ creatinine), while all other urinary amino acid levels were normal (see Table 1 ). This pattern of urinary aminoaciduria confirmed the diagnosis of DA. His brother (patient 1-II-2) was then screened and diagnosed at 40 years of age with DA, in light of isolated elevations in urinary glutamate and aspartate, with levels of 1,052 and $51 \mu \mathrm{mol} / \mathrm{mmol}$ creatinine, respectively. In proband 2 (patient 2-II-1), the urinary diagnosis for DA was confirmed at 4 years of age, with glutamate levels of 1,225 $\mu \mathrm{mol} / \mathrm{mmol}$ creatinine (normal range, $0-63 \mu \mathrm{mol} / \mathrm{mmol}$ creatinine) and aspartate levels of $50 \mu \mathrm{mol} / \mathrm{mmol}$ creatinine (normal range, $0-26 \mu \mathrm{mol} /$ mmol creatinine), with all other amino acids within their respective normal ranges. All other family members tested had urinary glutamate and aspartate levels within normal ranges.

We sequenced the candidate gene SLC1A1, based on its established role in glutamate and aspartate transport in rodents (6) and prior functional (19) and localization studies (5). All coding, untranslated, and regulatory regions of SLC1A1 were sequenced in genomic DNA from probands, including parents, siblings, and offspring (see Supplemental Table 1 for all primer sequences; supplemental material available online with this article; doi:10.1172/ JCI44474DS1). In pedigree 1, a missense mutation, c.1333C > T, in exon 12 of SLC1A1 was found, resulting in an arginine to tryptophan substitution at position 445 (p.R445W) (Figure 1A). Both affected siblings (patients 1-II-1 and 1-II-2) were homozygous for c.1333C > T, while all other family members were heterozygous (Table 2). In pedigree 2, a 3-bp deletion, c.1184-1186delTCA, was identified in exon 10 of SLC1A1, resulting in the deletion of isoleucine at position 395 (p.I395del) (Figure 1A). Proband 2 (patient 2-II-1) was homozygous for the c.1184-1186delTCA mutation, while the parents were both heterozygous (Table 2). Mutant SLC1A1 alleles in all homozygotes were consistent with an autosomal recessive inheritance pattern and segregated with the urinary phenotype. The inheritance of mutant alleles was independently confirmed by restriction fragment length polymorphism (RFLP) analysis in both pedigrees (Figure 1B). The c.1333C > T mutation was detected through the inactivation of an AgeI site, and c.1184-1186delTCA was detected through the creation of a BsaBI site. A panel of 480 normal Australian genomic DNA samples and an additional panel of 95 genomic DNA samples from FrenchCanadian subjects were subsequently screened by RFLP analysis to determine the allele frequency of both mutations. To the best of our knowledge, both mutations were novel and did not occur in their respective ethnically matched, normal populations. Interestingly, both mutations in SLC1A1 lie within a 39-kb region at chromosomal location 9p24 that has a significant association with OCD, based on a genome-wide linkage study (20), a replication study (21), and numerous family-based gene association studies (22-27) of early-onset OCD cohorts (summarized in Figure 1C).

Arginine 445, situated within transmembrane helix 8, is conserved in all known orthologs of SLC1A1 and within the 7 members of the SLC1 transporter family (Figure 2A). Isoleucine 395 is a conserved hydrophobic residue at identical positions in SLC1A1 orthologs and is the predominant residue in other SLC1 family members (Figure 2A). We mapped the predicted location of these residues, using the crystal structure of a prokaryotic glutamate transporter homolog from Pyrococcus horikoshii $\left(\mathrm{Glt}_{\mathrm{Ph}}\right)(28,29)$. R445 was pre- 
A

Pedigree 1

c. $1333 \mathrm{C}>\mathrm{T}$
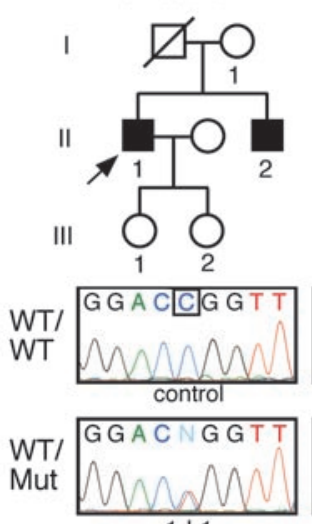

$1-1-1$

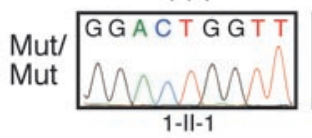

C

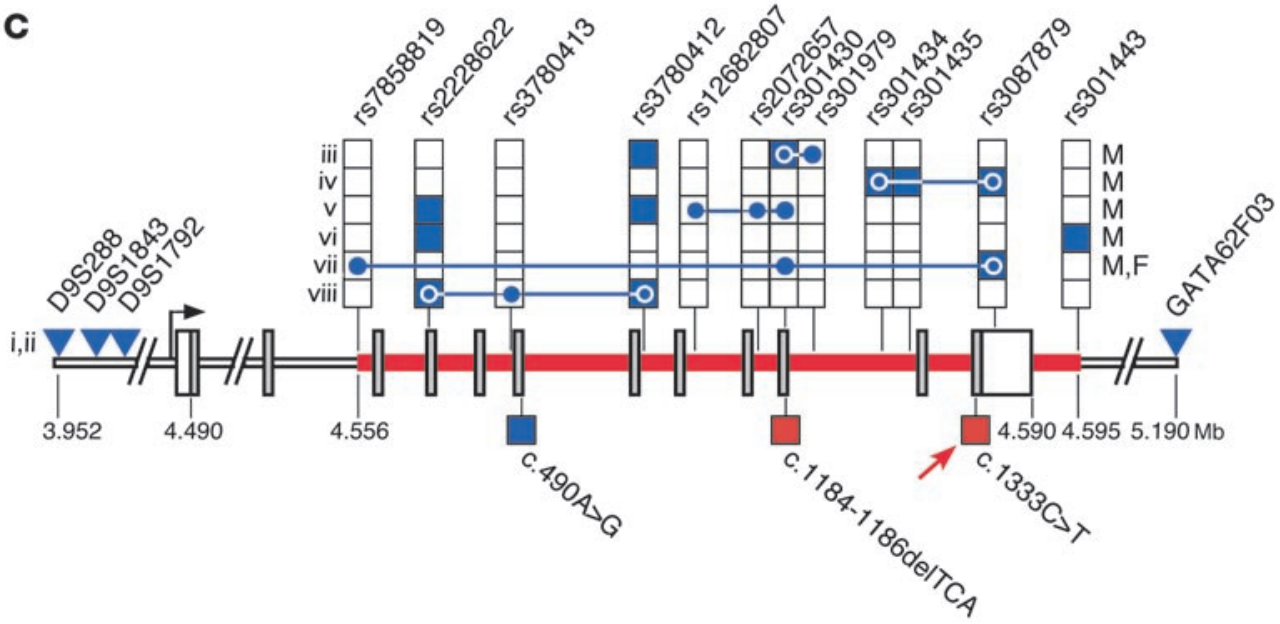

Figure 1

DA pedigrees with mutations identified in SLC1A1. (A) Pedigrees consistent with autosomal recessive inheritance are shown. Filled symbols represent individuals with DA, arrows indicate each proband, and the diagonal line represents a deceased subject. Representative chromatograms from control or DA pedigree DNA, with allele genotype (WT, wildtype; Mut, mutant) indicated. Boxes indicate the location of each mutation. (B) RFLP analysis of DA pedigrees. For c.1333C > T RFLP, Agel digestion releases 252- and 229-bp fragments (shown as control [C]). The c.1333C > T mutation inactivates the Agel site, leaving a 481-bp product (top panel). For c.1184-1186delTCA RFLP, BsaBI does not cut the 461-bp PCR product (shown as control). The c.1184-1186delTCA mutation results in a 458-bp amplicon, which is cut by $B s a B I$ into 245- and 213-bp fragments (bottom panel). L, 100-bp ladder. (C) The OCD-associated region at 9p24 containing SLC1A1. The 39-kb region identified in early-onset OCD cohorts is represented by the red line; SLC1A1 coding regions are represented by gray bars; untranslated regions are represented by vertical white bars; blue shading indicates a significant association with OCD $(P<0.05)$; red shading indicates an experimentally validated loss-of-function mutation; stacked boxes represent SNPs; blue triangles represent markers linked to OCD; connected blue circles indicate haplotype blocks significantly associated with OCD $(P<0.05)$; lower boxes indicate missense mutations (c.490A > G [T164A, ref. 40]), c.1184-1186delTCA [I395del], c.1333C > T [R445W]); red arrow indicates an association with OCD-like features; and coordinates represent telomeric distance (in $\mathrm{Mb}$ ). Sex-specific association with OCD is indicated (male [M] or female [F]). Roman numerals correlate with individual OCD studies: i (20); ii (21); iii (26); iv (27); v (25); vi (22); vii (23); and viii (24).

The functional effects of SLC1A1 mutations on substrate transport were examined in $X$. laevis oocytes. The addition of $100 \mu \mathrm{M}$ L-glutamate to oocytes injected with SLC1A1 WT complementary RNA (cRNA) generated an inward current (Figure 3A). This transport conductance decreased to $20 \%$ of that of WT in oocytes expressing SLC1A1 R445W mutant cRNA and was abrogated in oocytes expressing SLC1A1 I395del mutant cRNA (Figure 3A). Furthermore, the addition of excess L-glutamate $(1 \mathrm{mM})$ to oocytes expressing SLC1A1 I395del produced no transport current, similar to noninjected controls (Figure 3A). Glutamate washout from the R445W mutant transporter was considerably slower than that from WT (see current traces in Figure 3A). In oocytes expressing WT SLC1A1, the substrate concentration which elicits half the maximal transport current $\left(\mathrm{K}_{0.5}\right)$ for L-glutamate was $30 \pm 4 \mu \mathrm{M}$, while the $\mathrm{K}_{0.5}$ of L-glutamate-generated current for R445W was $2.0 \pm 0.3$ $\mu \mathrm{M}$ (Figure 3B). This apparent increase in L-glutamate affinity and slower washout of glutamate in R445W indicated that L-glutamate was more tightly bound compared with the WT transporter and may explain the reduced transporter currents observed. The rate of radiolabeled glutamate uptake in WTand R445W-expressing oocytes was examined at their respective $\mathrm{K}_{0.5}$ concentrations to accurately represent differences in glutamate transport activity. At $2 \mu \mathrm{M}^{3} \mathrm{H}$-L-glutamate the R445W mutant exhibited glutamate flux levels that were $2 \%$ of those of WT (Figure 3C). Again, the level of glutamate uptake in the I395del mutant was equivalent to that of noninjected oocytes, indicating that this transporter was nonfunctional (Figure 3C).

dicted to be in close proximity to the substrate-binding pocket, and I395 was predicted to be in HP2, a hairpin loop structure that has been shown to act as an extracellular gate governing access of the substrate to the binding site (Figure 2B) $(28,29)$.
The highly conserved $\mathrm{R} 445$ residue is in close proximity to an arginine residue (R447) that has been shown to interact with the $\gamma$-carboxyl group of glutamate (30) and an aspartate residue (D444), which is important for transport (Figure 2A and ref. 31). 
Table 2

Mutations identified in SLC1A1 in pedigrees with DA

\begin{tabular}{|c|c|c|c|}
\hline \multirow{3}{*}{$\begin{array}{l}\text { Gene } \\
\text { Locus } \\
\text { Allele }\end{array}$} & & \multicolumn{2}{|c|}{ SLC1A1 } \\
\hline & & $9 p 2$ & \\
\hline & & c.1184-1186delTCA & c. $1333 \mathrm{C}>\mathrm{T}$ \\
\hline Mutation & & p.1395del & p.R445W \\
\hline Patient & Phenotype & & \\
\hline $1-\mid-1$ & NM & $+/+$ & $+/-$ \\
\hline $1-\|-1$ & $\mathrm{DA}$ & $+/+$ & $-1-$ \\
\hline $1-\|-2$ & DA & $+/+$ & $-1-$ \\
\hline $1-|| \mid-1$ & NM & $+/+$ & $+/-$ \\
\hline $1-|| \mid-2$ & NM & $+/+$ & $+/-$ \\
\hline $2-I-1$ & NM & $+/-$ & $+/+$ \\
\hline $2-1-2$ & NM & $+/-$ & $+/+$ \\
\hline $2-I I-1$ & DA & $-1-$ & $+/+$ \\
\hline
\end{tabular}

Each patient is listed with the phenotype (either DA or normal [NM]) and with the informative genotype obtained from sequencing SLC1A1 (RefSeq NM_004170.4).

We and others have previously shown that mutation of R445 in SLC1A1 to serine, methionine, or glutamine or the equivalent arginine residue (R447) in SLC1A3 (EAAT1) to methionine results in low levels of glutamate flux and a large glutamate-activated leak cation conductance $(32,33)$. However, when R445 was mutated to the bulky, hydrophobic tryptophan residue present in pedigree 1 , glutamate flux levels were similarly reduced, but there was no glutamate-activated leak cation current (Supplemental Figure 1).

SLC1A1 is known to transport the glutathione precursor L-cysteine (34); however, subjects with DA do not hyperexcrete L-cysteine or its endogenous oxidation product L-cystine. The $\mathrm{K}_{0.5}$ values for L-cysteine transport measured from WT- and R445W-expressing oocytes were $115 \pm 13 \mu \mathrm{M}$ and $3 \pm 0.1 \mu \mathrm{M}$, respectively (Figure $3 \mathrm{D})$. The increased affinity for L-cysteine resulting from the $\mathrm{R} 445 \mathrm{~W}$ mutation in SLC1A1 led to a reduction in ${ }^{35} \mathrm{~S}$-L-cysteine uptake to levels that were $2 \%$ of those of WT, while I395del prevented ${ }^{35}$ S-L-cysteine uptake by SLC1A1 (Figure 3E). Consequently, the increase in L-glutamate and L-cysteine affinity for R445W not only reduced the size of the transporter currents observed but also substantially reduced the rate of substrate transport. Complementary to these functional studies, analysis of the surface expression of EGFP-tagged SLC1A1 in oocytes was performed. WT SLC1A1 was expressed uniformly on the oocyte surface (Figure $3 \mathrm{~F}$ ), while

\section{Figure 2}

Mapping of the SLC1A1 mutations. (A) Highly conserved regions in orthologs of SLC1A1 (top group) and SLC1 family members (middle group) and the SLC1A1 consensus sequence (bottom). Residues I395 and $\mathrm{R} 445$, deleted and mutated, respectively, in DA pedigrees are indicated. Residues known to be functionally important in SLC1A1, aspartate 444 (31) and arginine 447 (30), are indicated (diamonds). Blocks of the same color highlight groups of the same or similar amino acids: yellow, hydrophobic; red, acidic; orange, basic; purple, aromatic; blue, amido; green, hydroxyl; gray, proline; and pink, sulfur containing. (B) Predicted location of mutant residues based on the crystal structure of the prokaryotic glutamate transporter (Glt $\mathrm{Ph}_{\mathrm{Ph}}$ ). R445 (equivalent to M395 in Glt $t_{\mathrm{Ph}}$ ) is predicted to be located in transmembrane 8, and I395 (equivalent to I339 in Gltph) is in hairpin loop 2 (HP2) near the hinge region. Blue spheres indicate sodium ions; Asp represents the aspartate substrate. surface expression of the SLC1A1 R445W mutant was greatly reduced (Figure $3 \mathrm{G}$ ). Surface expression was not observed in noninjected oocytes (Figure 3H).

Expression studies were performed to ascertain whether these mutations also affected SLC1A1 surface expression in MadinDarby canine kidney (MDCKII) cells. In WT SLC1A1-expressing cells, expression was observed at the plasma membrane as well as in cytoplasmic reservoirs within the cell (Figure 4A) similar to that described in glioma cells (35). Introduction of the R445W mutation greatly decreased expression overall, with low level or absent membrane staining observed (Figure 4B), similar to our observation in oocytes. We have shown reduced or minimal in vitro surface expression of R445W, which would be the primary determinant of reduced or minimal glutamate and cysteine uptake (Figure $3 \mathrm{G}$ and Figure 4B). However, some residual surface expression of mutant SLC1A1 in vivo in humans may occur, and the reduced intrinsic impairment of transporter capacity (Figure 3, C and E) would further impair net substrate transport. The expression of the I395del mutant was negligible (Figure 4C) and similar to that of background levels (Figure 4D), supporting our evidence that the I395del mutation renders SLC1A1 nonfunctional.

In normal human kidney, SLC1A1 was distributed at the apical surface throughout the proximal tubule, including segment S1 emerging from the glomerulus (Figure 4E) and later segments S2 and S3 (data not shown), consistent with previous analysis in rodent kidney (5). The absence of apical staining in the peptide-blocked control confirmed antibody specificity (Figure 4H). SLC1A1 also colocalized with Lotus tetragonolobus agglutinin (LTA) binding, the known apical marker of proximal tubules

A
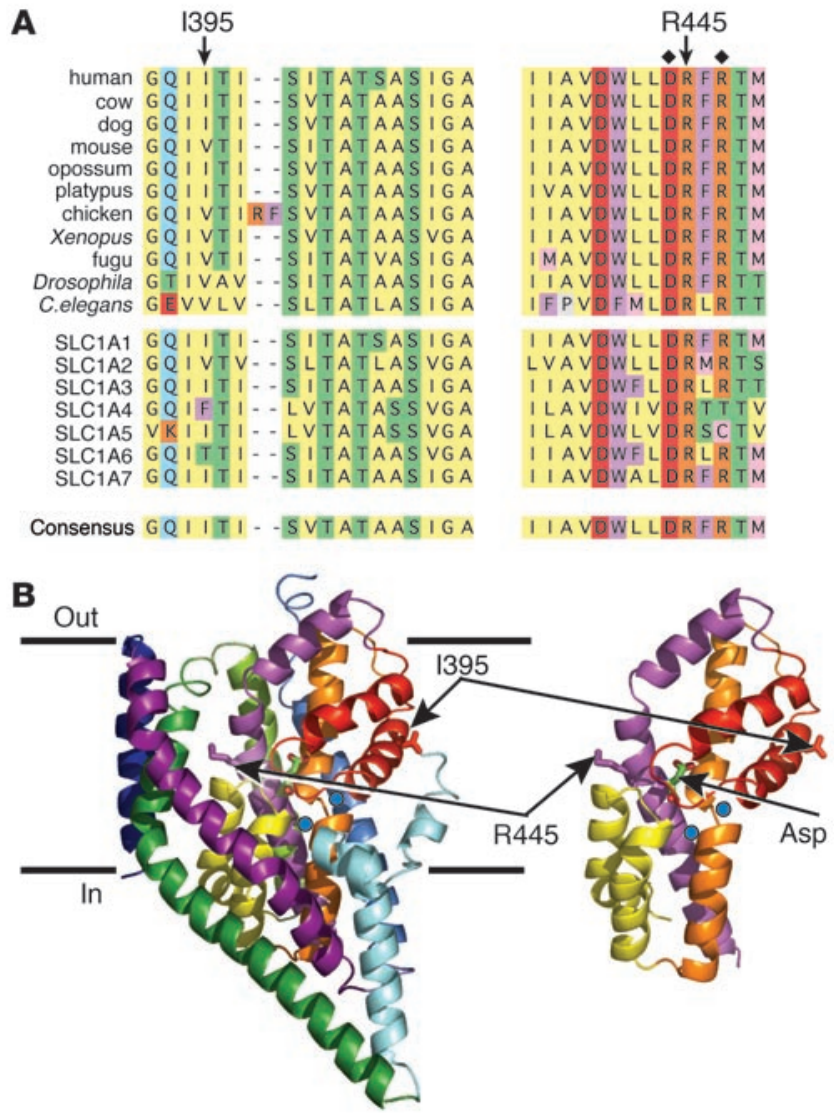

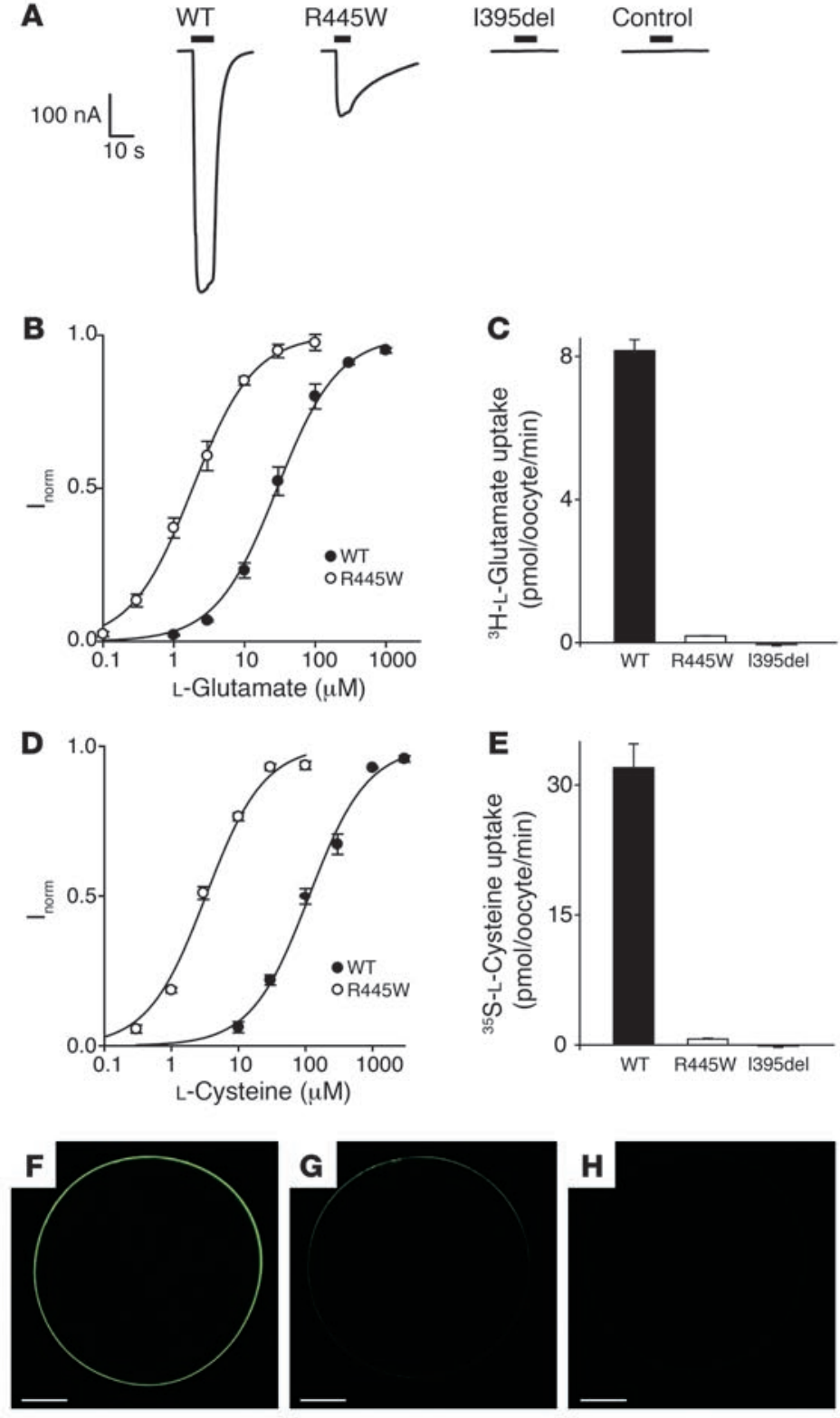

\section{Figure 3}

Mutations in SLC1A1 from subjects with DA affect its function. (A) Representative oocytes expressing WT, R445W, or I395del CRNA or noninjected oocytes (control) were clamped at $-60 \mathrm{mV}$ and perfused with ND96 buffer containing $100 \mu \mathrm{M}$ (for WT and $\mathrm{R} 445 \mathrm{~W}$ ) or $1 \mathrm{mM}$ (for I395del and control) L-glutamate. Black scale bars represent the time (s, $x$ axis) that L-glutamate was applied and the current size (nA, $y$ axis). Scale is shown for time versus current. (B) The L-glutamate dose response for WT (filled circles) and R445W (open circles). Current ( $I_{\text {norm }}$ ) was normalized to the maximal current, defined as unit $I_{\max }\left(\mathrm{WT}, \mathrm{K}_{0.5}=30 \pm 4\right.$ $\mu \mathrm{M}$ and $I_{\max }=424 \pm 13 \mathrm{nA} ; \mathrm{R} 445 \mathrm{~W}, \mathrm{~K}_{0.5}=2 \pm 0.3 \mu \mathrm{M}$ and $I_{\max }$ $53 \pm 7 \mathrm{nA}$ ). (C) Radiolabeled L-glutamate uptake at $\mathrm{K}_{0.5}$. (D) The L-cysteine dose response for WT (filled circles) and R445W (open circles). Current was normalized to the maximal current $\left(\mathrm{WT}, \mathrm{K}_{0.5}=115 \pm 13 \mu \mathrm{M}\right.$ and $\mathrm{I}_{\max }=590 \pm 19 \mathrm{nA} ; \mathrm{R} 445 \mathrm{~W}$, $\mathrm{K}_{0.5}=3.3 \pm 0.1 \mu \mathrm{M}$ and $\mathrm{I}_{\max }=54 \pm 20 \mathrm{nA}$ ). (E) Radiolabeled L-cysteine uptake at $\mathrm{K}_{0.5} \cdot{ }^{3} \mathrm{H}$-L-glutamate and ${ }^{35} \mathrm{~S}$-L-cysteine uptake in noninjected oocytes was subtracted from the data presented ( $\mathbf{C}$ and $\mathbf{E}$, respectively). All data represent the mean \pm SEM of at least 3 oocytes (in B-E). (F-H) Representative oocytes expressing EGFP-tagged SLC1A1 cRNA. (F) WT oocytes. (G) R445W oocytes. (H) Noninjected control oocytes. Scale bar: $200 \mu \mathrm{m}$.

cally matched populations; third, they occurred in regions of SLC1A1 that are functionally important for substrate transport; fourth, they nearly or fully abolished substrate transport and cell surface expression; and fifth, the distribution of SLC1A1 in the proximal tubule was consistent with a primary role for high-affinity uptake of glutamate and aspartate in the human kidney. In subjects with no residual SLC1A1 function an isolated DA was evident. These data establish that SLC1A1 is responsible for the majority, if not all, of renal glutamate and aspartate reabsorption. In contrast, the majority of renal cysteine transport occurs in the oxidized state as cystine, facilitated by a heterodimeric exchanger, comprising SLC3A1 and SLC7A9, and thus can still occur when SLC1A1 is inactivated. As is the case for renal autosomal recessive disorders, such as Hartnup disorder $(16,17)$ and iminoglycinuria (18), one functional allele suffices to prevent urinary wastage of amino acids.

Our findings on SLC1A1 disruption in humans were in agreement with the DA phenotype reported in Slc1a1 nullizygous mice (6). Despite this phenotypic similarity between

(Figure 4, F and G). Overall, the distribution of SLC1A1 was consistent with its major role in high-affinity glutamate reabsorption in the kidney. A summary of our findings is depicted in a model of the renal proximal tubule (Figure 5). The principle amino acid transporters involved in amino acid reabsorption, which have been identified by us and others, to be mutated in inherited human aminoacidurias are now all shown.

\section{Discussion}

Until now definitive proof of the involvement of SLC1A1 in human DA was lacking despite prior speculation $(6,36)$. We report 2 mutations, a missense mutation, R445W, and a deletion, I395del, in the high-affinity glutamate transporter SLC1A1 from 2 independent pedigrees with DA. Our molecular and functional analyses of these SLC1A1 mutations provide evidence for their involvement in the pathogenesis of DA. First, both mutations segregated with the urinary phenotype; second, they were not present in normal, ethnispecies, the appearance of age-related neurodegeneration, which was observed in outbred Slc1a1 nullizygous mice (9), has not yet been clearly documented in humans. This neurodegeneration was attributed to decreased neuronal cysteine uptake (9). Cysteine is the rate-limiting substrate for the synthesis of glutathione, and neurons are dependent on the extracellular uptake of cysteine for normal function. Most cell types transport cysteine in the form of cystine, by heteroexchange with glutamate via the sodium-independent system $\mathrm{x}_{\mathrm{c}}^{-}$(37), but in vitro studies suggest that neurons lose this capacity as they mature (38). Neurons use sodium-dependent cysteine uptake as the major route for cysteine uptake (80\%-90\% of total uptake), most of which is handled by SLC1A1 (75\%-85\% of total sodium-dependent uptake) (39). Thus, abrogation of cysteine uptake in the neurons of outbred Slc1a1 nullizygous mice led to impaired glutathione metabolism, increased oxidative stress, and neuronal death (9). However, in a separate study using Slc1a1 nullizygous mice that were not fully backcrossed to the inbred C57BL/6 

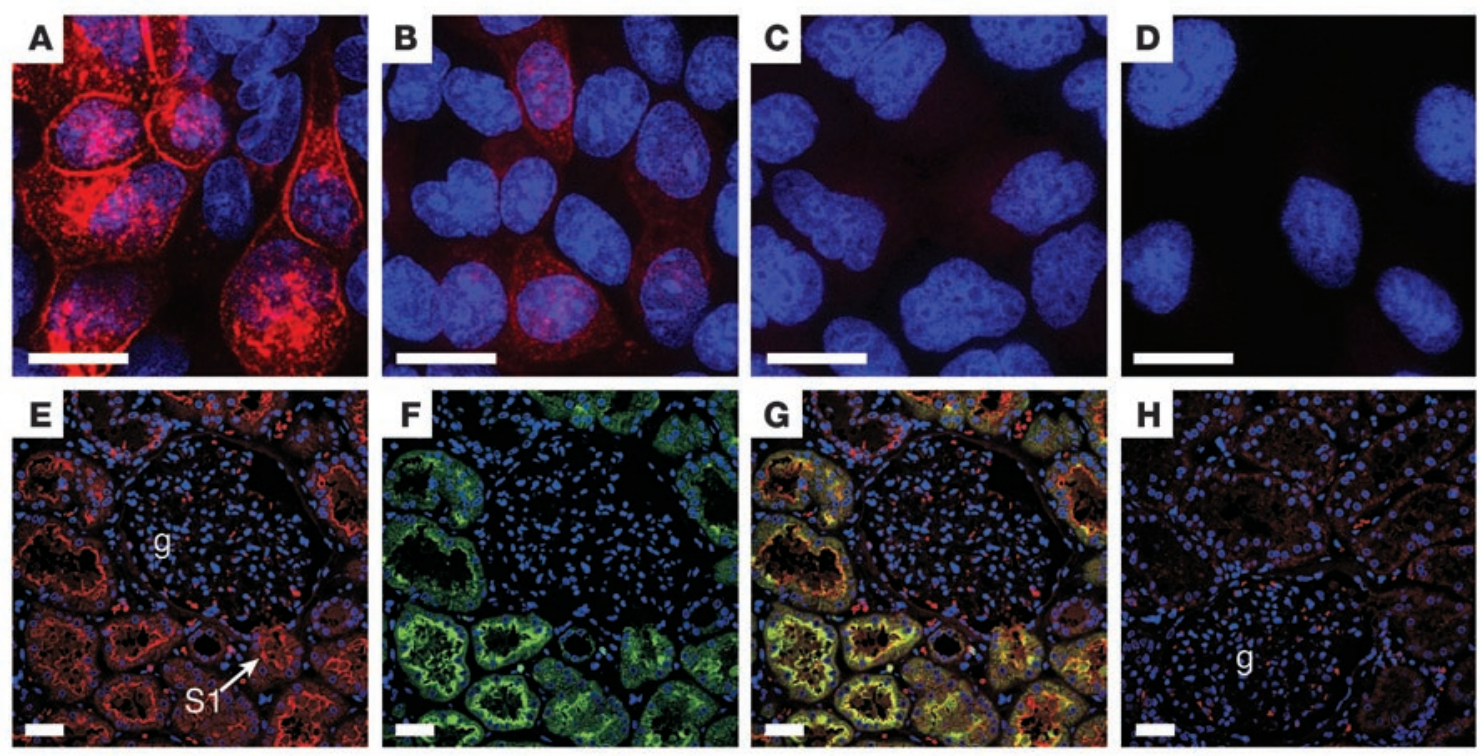

\section{Figure 4}

Tissue and subcellular distribution of SLC1A1. (A-D) Immunofluorescence of hemagglutinin epitope-tagged WT and mutant cDNAs stably expressed in MDCKII cells (A) SLC1A1 WT, (B) SLC1A1 R445W, and (C) SLC1A1 I395del MDCKII, and (D) empty vector (pcDNA3.1-HA). Scale bar: $10 \mu \mathrm{m}$. (E-H) Immunofluorescence of normal adult human kidney, showing SLC1A1 expression in proximal tubules. Kidney sections were stained with an SLC1A1 antibody (red) and the proximal tubule marker LTA (green). Nuclei were stained with DAPI (blue). (E) SLC1A1 expression on the apical membrane of kidney tubules. An example of an S1 segment (arrow) emerging from the glomerulus (g) is indicated. (F) LTA staining identifying the apical membrane of proximal tubules. (G) Colocalization (yellow) of SLC1A1 and LTA on the apical membrane of S1 segments of the proximal tubule (confocal images in $\mathbf{E}$ and $\mathbf{F}$ were merged). (H) Peptide-blocked control. A glomerulus is indicated. Scale bar: $30 \mu \mathrm{m}$.

strain (N4-N7), indicators of oxidative stress in young adult mice were not detected (10). This indicates that the genetic background of the Slc1a1 nullizygous mice will be important when interpreting any future neurological or behavioral studies. SLC1A1 function in renal epithelial cells is readily detected by urine analysis; however, evidence of reduced or absent SLC1A1 function in human neurons is more difficult to demonstrate.

Neurological dysfunction such as mental retardation has been associated with DA in 2 independent clinical reports (2, 3); however, due to the small number of clinically evaluable individuals with DA and the potential age-related onset of symptoms, neurological assessment has been difficult. Intriguingly, proband 1 in pedigree 1 exhibited features consistent with a diagnosis of OCD, but he declined formal psychological assessment for OCD. This observation is interesting in light of the strong association between SLC1A1 and OCD, as revealed by genome-wide linkage (20), replication (21), and family-based association studies (22-27) of early-onset OCD cohorts in which a genetic contribution to disease etiology is likely. Furthermore, 5 out of 6 family-based gene association studies showed a significant association of polymorphisms or haplotypes in SLC1A1 to early-onset OCD in males. OCD is characterized by increased behavioral and cognitive rigidity and a loss of executive control over actions as well as dysfunction in associated neural circuitry. Slc1a1 nullizygous mice have not yet been thoroughly examined for executive dysfunction phenotypical of OCD. To date, only one rare variant, c.490A > G (p.T164A), in a conserved region of SLC1A1 has been identified in OCD cohorts (40), though this has not been functionally tested. A molecular explanation for a role for $S L C 1 A 1$ in OCD may yet lie in noncoding regions within or immediately adjacent to the SLC1A1 locus.
In this report we have demonstrated that recessive mutations in SLC1A1 lead to DA. This discovery provides what we believe to be the only known human examples for the loss of function of SLC1A1 and demonstrates its major role in renal glutamate and aspartate handling in humans. The extent of glutamate and cysteine transport non-redundancy in neurons due to mutations in SLC1A1 in human DA patients, and therefore neurological dysfunction, may entirely depend on the degree of loss of function and patient age. Neurodegeneration may result only when SLC1A1mediated cysteine transport is fully abrogated, as observed in Slc1a1 nullizygous mice. The same SLC1A1-mediated defect in renal glutamate transport may provoke abnormal neuronal function in a subset of patients with DA.

\section{Methods}

Sample collection and analysis. Institutional ethics committee approval was obtained for all studies in humans (University of Sherbrooke Health Centre, 05-005 and 05-005R, and Sydney South West Area Health Service, X040239 and X06-0172). All adult subjects provided written informed consent, and for proband 2, informed consent was obtained from both parents. Quantitative urinary amino acid analysis of morning urine samples was performed by ion exchange chromatography, as we have described $(18,41)$. DNA was isolated from saliva samples collected using self-collection kits (DNAgenotek) or from peripheral blood mononuclear cells (PBMCs) isolated by Ficoll density gradient centrifugation. PBMCs were immortalized by Epstein-Barr virus transformation, as we have described (17). The panel of Australian control genomic DNA samples (18) was isolated from blood samples from 480 deidentified individuals collected during routine testing. The panel of 95 French-Canadian control DNAs represents a group of patients collected for the purpose of association studies in various neuro- 


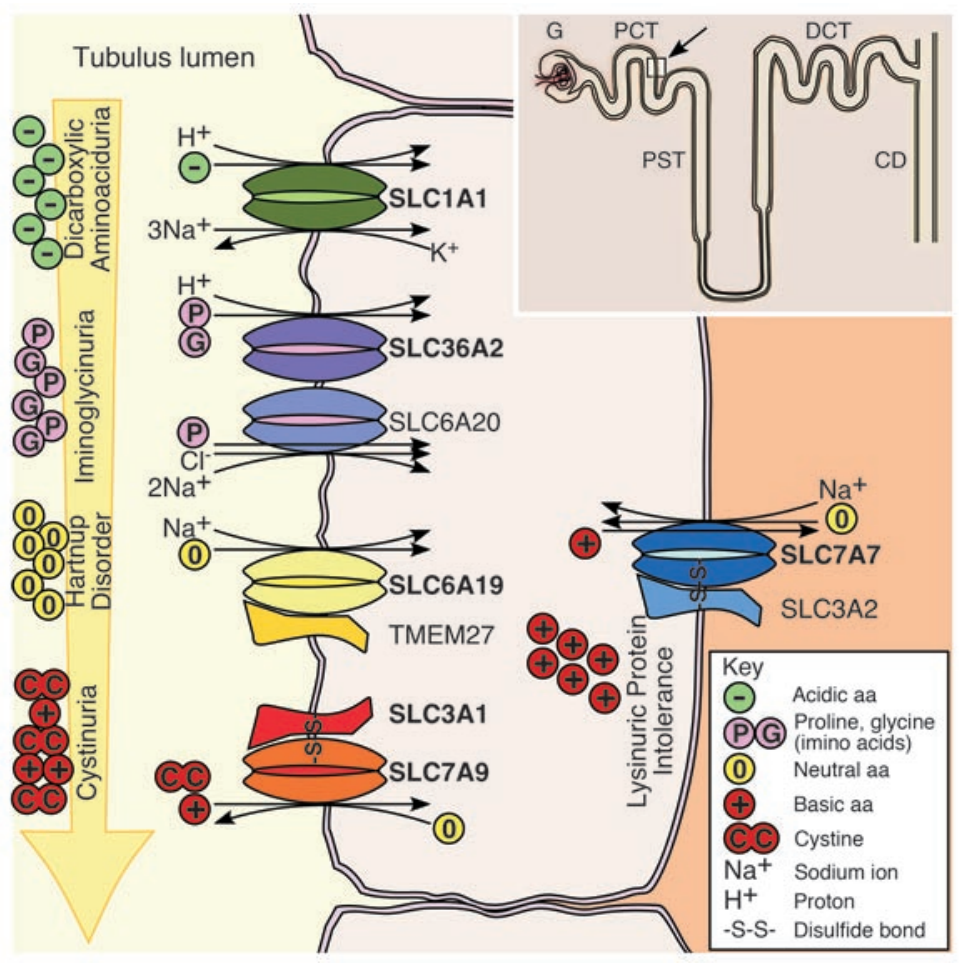

Figure 5

A model of the renal proximal tubule, illustrating the principal epithelial transporters involved in amino acid reabsorption, which are mutated in human aminoacidurias. A nephron is depicted (inset), showing the glomerulus, proximal convoluted tubule (PCT), proximal straight tubule (PST), distal convoluted tubule (DCT), and collecting duct $(C D)$. A cross-section of the proximal convoluted tubule (white square indicated with an arrow) is represented in the main diagram. Four of the aminoacidurias, including DA, iminoglycinuria, Hartnup disorder, and cystinuria manifest at the apical surface of the renal tubule, while lysinuric protein intolerance manifests at the basolateral surface. Mutations in the high-affinity glutamate and aspartate transporter SLC1A1, responsible for DA, were identified in this study. Iminoglycinuria results from complete inactivation of SLC36A2, a proline and glycine transporter, or from additional modifying mutations in the high-affinity proline transporter SLC6A20 when SLC36A2 is not completely inactivated (18). Mutations in the neutral amino acid transporter, SLC6A19, are responsible for Hartnup disorder $(16,17)$. The neutral amino acid transport defect can also be exacerbated by a kidney-specific loss of heterodimerization of mutant SLC6A19 with TMEM27 (44). Cystinuria has a heterogeneous phenotype and arises from mutations in individual or both subunits of the disulfide bridge-linked heterodimer comprising the type II membrane protein SLC3A1 (12) and the cystine and basic amino acid transporter SLC7A9 $(13)$. Lysinuric protein intolerance $(14,15)$ results from mutations in the basolaterally expressed basic amino acid transporter SLC7A7, which forms a disulfide bridge-linked heterodimer with type II membrane protein SLC3A2. The major transporters involved in each aminoaciduria are in bold. For a detailed review of renal epithelial amino acid transport systems and their involvement in disease see Bröer (45).

logical disorders (obtained from Jean-Baptiste Rivière and Guy Rouleau, University of Montreal, Montreal, Quebec, Canada). They have an average age of 60, are all from the Quebec province, and the 4 grandparents of each individual were all born in Quebec. A neurologist has examined them all, and no apparent neurological disorder was observed.

Molecular analysis. Nucleotide coordinates for the SLC1A1 mutations were taken from the RefSeq mRNA sequence for SLC1A1 (NM_004170.4). SLC1A1 c.1184-1186delTCA (p.I395del) was identified by a RFLP resulting from the creation of a BsaBI restriction enzyme site, and c.1333C > T (p.R445W) was determined by an RFLP resulting from inactivation of an AgeI restriction enzyme site (see Supplemental Table 1 for primer sequences). The SLC1A1 cDNA was amplified using RT-PCR from human kidney mRNA (BD Biosciences) or from mRNA isolated from immortalized PBMCs from proband 1 and cloned into pGEMT-Easy (Promega). The SLC1A1 open reading frame was PCR amplified from pGEM-T-SLC1A1 as a 1,793-bp product incorporating EcoRI ends and then cloned into the mammalian expression vector pcDNA3.1-HA (18) for N-terminal hemagglutinin epitope tagging. The R445W mutant was excised from pGEM-T-SLC1A1(R445W) with BstEII/AflII and ligated into pcDNA3.1-HA-SLC1A1. The I395del mutation was synthesized as a 424-bp fragment comprising c.924-1350, incorporating the c.1184-1186delTCA deletion, and provided in the pJ201 plasmid (DNA2.0). A 410-bp BsiWI/ AgeI fragment containing the I395del mutation was ligated into pcDNA3.1-HA-SLC1A1. For functional studies in X. laevis oocytes, SLC1A1 was PCR amplified with KpnI/BamHI ends and ligated into the oocyte expression vector pOTV (19) cut with KpnI/BglII. I395del and R445W mutant vectors were constructed by cloning the 410-bp BsiWI/AgeI I395del fragment and a 213-bp AccI R445W fragment into pOTV-SLC1A1, respectively. For surface expression in oocytes, EGFP was fused to the C terminus of SLC1A1. All protein sequences used in alignments were obtained from Ensembl release 57 and aligned using the ClustalW algorithm. Where sequence identity was $50 \%$ or greater, the conserved residue was indicated.

Functional analysis. Radiolabeled glutamate and cysteine uptake and electrophysiological studies were performed in $X$. laevis oocytes after injection of in vitro-transcribed cRNA as previously described $(34,42,43)$. Substrate uptake was measured by placing 5 oocytes in ND96 buffer containing ${ }^{3} \mathrm{H}$-L-glutamate (GE Healthcare) or ${ }^{35} \mathrm{~S}$-L-cysteine (Perkin Elmer). ${ }^{35} \mathrm{~S}$-L-cysteine was stored at $-80^{\circ} \mathrm{C}$ in $5 \mathrm{mM} \mathrm{DTT}$ under nitrogen, and all solutions were freshly prepared prior to use to minimize oxidation of cysteine.

Subcellular localization and tissue staining. Immunofluorescence of pcDNA3.1-HA-SLC1A1-transfected MDCKII cells and deidentified normal human kidney samples was performed as previously described (18). SLC1A1 expression was detected with a polyclonal goat anti-SLC1A1 antibody (1:10; sc-7761, Santa Cruz Biotechnology Inc.) raised against a peptide corresponding to the $\mathrm{C}$ terminus (sc-7761P, Santa Cruz Biotechnology Inc.) and a donkey anti-goat Alexa Fluor 594 (1:400; Invitrogen). Proximal tubules were marked using the biotinylated lectin LTA (1:100; Vector Laboratories) and detected with streptavidin-Alexa Fluor 488 (Invitrogen). All antibodies were diluted in PBS. Digital immunofluorescence images of cell and tissue staining were obtained with identical gain settings using a $\times 40$ oil objective installed on a Leica SP5 confocal microscope, using the 405-nm and 561-nm lasers. Oocytes expressing EGFP-tagged SLC1A1 were imaged live with a $\times 10$ air objective using the 405-nm laser. All image series were equally processed using Canvas X (ACD Systems International).

Statistics. All functional studies in Xenopus oocytes were assessed using 2-way ANOVA. Mutant transporter activity in all cases was significantly different to that of WT $(P<0.001)$.

\section{Acknowledgments}

Financial support was provided by the Australian National Health and Medical Research Council (NHMRC; project grant 402730), the Australian Research Council (Discovery Project DP0877897), University of Sydney Bridging Support Grant (RIMS 2009-02579), the Rebecca L. Cooper Foundation, and Cure The Future Founda- 
tion. R.M. Ryan is supported by an NHMRC Career Development Award, and R.J. Vandenberg is supported by an NHMRC Senior Research Fellowship. Assistance in clinical amino acid quantitation was provided by Robert Giguère from the Biochemical Genetics Service, Centre Hospitalier Université de Sherbrooke and Kevin Carpenter of the New South Wales Biochemical Genetics and Newborn Screening Services at The Children's Hospital at Westmead. We thank Mairwen Jones for helpful discussions, Cheryl Handford for assistance in oocyte preparation, Angelika Bröer for oocyte transport assays, and Jean-Baptiste Rivière and Guy Rouleau of the Centre of Excellence in Neuromics, University of Montreal, for normal, French-Canadian human genomic DNA samples.

Received for publication July 22, 2010, and accepted in revised form October 20, 2010.

Address correspondence to: John E.J. Rasko, Centenary Institute, Locked Bag No. 6, Newtown 2042, New South Wales, Australia. Phone: 61.2.95656156; Fax: 61.2.95656101; E-mail: j.rasko@ centenary.org.au.
1. Auray-Blais C, Cyr D, Drouin R. Quebec neonatal mass urinary screening programme: from micromolecules to macromolecules. J Inherit Metab Dis. 2007; 30(4):515-521.

2. Teijema HL, van Gelderen HH, Giesberts MA, Laurent de Angulo MS. Dicarboxylic aminoaciduria: an inborn error of glutamate and aspartate transport with metabolic implications, in combination with a hyperprolinemia. Metabolism. 1974;23(2):115-123.

3. Swarna M, Rao DN, Reddy PP. Dicarboxylic aminoaciduria associated with mental retardation. Hum Genet. 1989;82(3):299-300.

4. Kanai Y, Hediger MA. Primary structure and functional characterization of a high-affinity glutamate transporter. Nature. 1992;360(6403):467-471.

5. Shayakul C, Kanai Y, Lee WS, Brown D, Rothstein JD, Hediger MA. Localization of the high-affinity glutamate transporter EAAC1 in rat kidney. $A m J$ Physiol. 1997;273(6 pt 2):F1023-F1029.

6. Peghini P, Janzen J, Stoffel W. Glutamate transporter EAAC-1-deficient mice develop dicarboxylic aminoaciduria and behavioral abnormalities but no neurodegeneration. EMBOJ. 1997;16(13):3822-3832.

7. Rothstein JD, Van Kammen M, Levey AI, Martin LJ, Kuncl RW. Selective loss of glial glutamate transporter GLT-1 in amyotrophic lateral sclerosis. Ann Neurol. 1995;38(1):73-84.

8. Nieoullon A, Canolle B, Masmejean F, Guillet B, Pisano $\mathrm{P}$, Lortet $\mathrm{S}$. The neuronal excitatory amino acid transporter EAAC1/EAAT3: does it represent a major actor at the brain excitatory synapse? J Nenrochem. 2006;98(4):1007-1018.

9. Aoyama K, et al. Neuronal glutathione deficiency and age-dependent neurodegeneration in the EAAC1 deficient mouse. Nat Neurosci. 2006;9(1):119-126.

10. Scimemi A, Tian H, Diamond JS. Neuronal transporters regulate glutamate clearance, NMDA receptor activation, and synaptic plasticity in the hippocampus. J Neurosci. 2009;29(46):14581-14595.

11. Camargo SM, Bockenhauer D, Kleta R. Aminoacidurias: Clinical and molecular aspects. Kidney Int. 2008;73(8):918-925.

12. Calonge MJ, et al. Cystinuria caused by mutations in $\mathrm{rBAT}$, a gene involved in the transport of cystine. Nat Genet. 1994;6(4):420-425.

13. Feliubadaló L, et al. Non-type I cystinuria caused by mutations in SLC7A9, encoding a subunit (bo,+AT) of rBAT. Nat Genet. 1999;23(1):52-57.

14. Torrents D, et al. Identification of SLC7A7, encod-

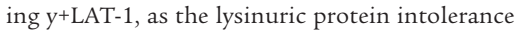
gene. Nat Genet. 1999;21(3):293-296.

15. Borsani G, et al. SLC7A7, encoding a putative permease-related protein, is mutated in patients with lysinuric protein intolerance. Nat Genet. 1999; 21(3):297-301.

16. Kleta R, et al. Mutations in SLC6A19, encoding B0AT1, cause Hartnup disorder. Nat Genet. 2004;
36(9):999-1002.

17. Seow HF, et al. Hartnup disorder is caused by mutations in the gene encoding the neutral amino acid transporter SLC6A19. Nat Genet. 2004; 36(9):1003-1007.

18. Bröer $\mathrm{S}$, et al. Iminoglycinuria and hyperglycinuria are discrete human phenotypes resulting from complex mutations in proline and glycine transporters. J Clin Invest. 2008;118(12):3881-3892.

19. Arriza JL, Fairman WA, Wadiche JI, Murdoch GH, Kavanaugh MP, Amara SG. Functional comparisons of three glutamate transporter subtypes cloned from human motor cortex. J Neurosci. 1994; 14(9):5559-5569.

20. Hanna GL, et al. Genome-wide linkage analysis of families with obsessive-compulsive disorder ascertained through pediatric probands. Am J Med Genet. 2002;114(5):541-552.

21. Willour VL, et al. Replication study supports evidence for linkage to $9 \mathrm{p} 24$ in obsessive-compulsive disorder. Am J Hum Genet. 2004;75(3):508-513.

22. Shugart YY, et al. A family-based association study of the glutamate transporter gene SLC1A1 in obsessive-compulsive disorder in 378 families. Am J Med Genet B Neuropsychiatr Genet. 2009;150B(6):886-892.

23. Wendland JR, et al. A haplotype containing quantitative trait loci for SLC1A1 gene expression and its association with obsessive-compulsive disorder. Arch Gen Psychiatry. 2009;66(4):408-416.

24. Kwon JS, et al. Association of the glutamate transporter gene SLC1A1 with atypical antipsychoticsinduced obsessive-compulsive symptoms. Arch Gen Psychiatry. 2009;66(11):1233-1241.

25. Stewart SE, et al. Association of the SLC1A1 glutamate transporter gene and obsessive-compulsive disorder. Am J Med Genet B Neuropsychiatr Genet. 2007;144B(8):1027-1033.

26. Dickel DE, et al. Association testing of the positional and functional candidate gene SLC1A1/EAAC1 in early-onset obsessive-compulsive disorder. Arch Gen Psychiatry. 2006;63(7):778-785.

27. Arnold PD, Sicard T, Burroughs E, Richter MA, Kennedy JL. Glutamate transporter gene SLC1A1 associated with obsessive-compulsive disorder. Arch Gen Psychiatry. 2006;63(7):769-776.

28. Boudker O, Ryan RM, Yernool D, Shimamoto K, Gouaux E. Coupling substrate and ion binding to extracellular gate of a sodium-dependent aspartate transporter. Nature. 2007;445(7126):387-393.

29. Yernool D, Boudker O, Jin Y, Gouaux E. Structure of a glutamate transporter homologue from Pyrococcus horikoshii. Nature. 2004;431(7010):811-818.

30. Bendahan A, Armon A, Madani N, Kavanaugh MP, Kanner BI. Arginine 447 plays a pivotal role in substrate interactions in a neuronal glutamate transporter. J Biol Chem. 2000;275(48):37436-37442.

31. Teichman S, Kanner BI. Aspartate- 444 is essential for productive substrate interactions in a neuronal glutamate transporter.J Gen Physiol. 2007;129(6):527-539.

32. Ryan RM, Kortt NC, Sirivanta T, Vandenberg RJ. The position of an arginine residue influences substrate affinity and $\mathrm{K}(+)$ coupling in the human glutamate transporter, EAAT1. J Neurochem. 2010; 114(2):565-575.

33. Borre L, Kanner BI. Arginine 445 controls the coupling between glutamate and cations in the neuronal transporter EAAC-1. J Biol Chem. 2004; 279(4):2513-2519.

34. Zerangue N, Kavanaugh MP. Interaction of L-cysteine with a human excitatory amino acid transporter. J Physiol. 1996;493(pt 2):419-423.

35. Fournier KM, González MI, Robinson MB. Rapid trafficking of the neuronal glutamate transporter, EAAC1: evidence for distinct trafficking pathways differentially regulated by protein kinase $\mathrm{C}$ and platelet-derived growth factor. J Biol Chem. 2004; 279(33):34505-34513.

36. Smith CP, Weremowicz S, Kanai Y, Stelzner M, Morton CC, Hediger MA. Assignment of the gene coding for the human high-affinity glutamate transporter EAAC1 to 9p24: potential role in dicarboxylic aminoaciduria and neurodegenerative disorders. Genomics. 1994;20(2):335-336.

37. Wu G, Fang YZ, Yang S, Lupton JR, Turner ND. Glutathione metabolism and its implications for health. J Nutr. 2004;134(3):489-492.

38. Murphy TH, Schnaar RL, Coyle JT. Immature cortical neurons are uniquely sensitive to glutamate toxicity by inhibition of cystine uptake. FASEBJ. 1990; 4(6):1624-1633.

39. Shanker G, Allen JW, Mutkus LA, Aschner M. The uptake of cysteine in cultured primary astrocytes and neurons. Brain Res. 2001;902(2):156-163.

40. Wang Y, et al. A screen of SLC1A1 for OCD-related alleles. Am J Med Genet B Neuropsychiatr Genet. 2010; $153 \mathrm{~B}(2): 675-679$

41. Auray-Blais C, Giguère R, Lemieux B. Newborn urine screening programme in the province of Quebec: an update of 30 years' experience. J Inherit Metab Dis. 2003;26(4):393-402.

42. Huang S, Ryan RM, Vandenberg RJ. The role of cation binding in determining substrate selectivity of glutamate transporters. J Biol Chem. 2009; 284(7):4510-4515.

43. Ryan RM, Mitrovic AD, Vandenberg RJ. Site-directed mutagenesis in the study of membrane transporters. Methods Mol Biol. 2003;227:97-108.

44. Kowalczuk S, Bröer A, Tietze N, Vanslambrouck JM, Rasko JE, Bröer S. A protein complex in the brush-border membrane explains a Hartnup disorder allele. FASEB J. 2008;22(8):2880-2887.

45. Broer S. Amino acid transport across mammalian intestinal and renal epithelia. Physiological Reviews. 2008;88(1):249-286. 